\title{
High-resolution visible spectroscopy of the jet-driving star Th $28^{\star}$
}

\author{
F. Comerón ${ }^{1}$ and M. Fernández ${ }^{2}$
}

\author{
1 ESO, Karl-Schwarzschild-Strasse 2, 85748 Garching bei München, Germany \\ e-mail: fcomeron@eso.org \\ 2 Instituto de Astrofísica de Andalucía, CSIC, Camino Bajo de Huétor 50, 18008 Granada, Spain \\ e-mail: matilde@iaa.es
}

Received 22 July 2009 / Accepted 25 November 2009

\begin{abstract}
Context. We present a study of the extreme T Tauri star Th 28, a young stellar object in the Lupus 3 cloud whose spectrum displays all the varieties of signposts associated with early stellar activity. Th 28 is the driving source of a fast jet, making it a very promising target to study the disk-jet connection.

Aims. We try to identify and investigate the different structural components that contribute to the different emission lines in the spectrum of the Th 28 central source.

Methods. We obtained high-resolution visible spectroscopy with the UVES spectrograph at the VLT, using the resolved profiles of both permitted and forbidden lines as tracers of gas with different kinematic and physical properties, complemented with other observations from the literature.

Results. We identify four distinct structural components that contribute to the visible emission-line spectrum of Th 28 . The first one, dominating most of the permitted and forbidden lines, is probably associated with the origin of the outflow that in the past produced the Herbig-Haro objects seen to the west of Th 28. The second one is an uncollimated stellar wind characterized by high excitation and temperature, as shown by the broad profile of the intense [OIII] lines. The third component, traced only by permitted lines, appears as a redshifted tail extending up to radial velocities of $+450 \mathrm{~km} \mathrm{~s}^{-1}$, which we attribute to magnetospheric accretion. From the latter component we obtain a rough estimate of $0.6-0.9 M_{\odot}$ for the mass of the central object. Using published equivalent widths of the CaII triplet lines, we estimate an accretion rate of 4.2-6.3 $\times 10^{-8} M_{\odot} \mathrm{yr}^{-1}$, comparable to the values inferred for other T Tauri stars of similar mass. The last component, which appears most clearly in the [SII] lines and is hardly seen in any lines other than those of [OI], displays signatures that we interpret as coming from rotation, perhaps formed in a disk atmosphere. Following this interpretation, we estimate a disk central hole of 0.7 AU radius.

Conclusions. Even though spatially unresolved, the spectrum of Th 28 is rich in emission lines whose shapes provide important information on the distinct structures where they form. In this way we were able to identify the existence of a stellar wind, an accretion flow, the basis of a collimated outflow, and possibly a rotating disk atmosphere, obtaining rough estimates of basic parameters of the central source and its environment that should be useful for further modeling.
\end{abstract}

Key words. stars: formation - stars: pre-main sequence - stars: mass-loss - stars: individual: Th 28, Sz 102, Krautter's star

\section{Introduction}

The circumstellar environment of $\mathrm{T}$ Tauri stars is complex. Within a few astronomical units of the newly formed star, an array of the structures that accompany the early stages of stellar evolution (circumstellar disks, accretion flows, jets, stellar and disks winds, etc.) sample a wide range of physical and kinematical conditions that reveal themselves at all wavelengths from $\mathrm{X}$-rays to radio. Their spectral signatures overlap in a way that often challenges interpretation, with further complications coming from the way in which they change with viewing geometry or with the effects of obscuring dust and opaque disks. While their interpretation is difficult, those spectral signatures carry very valuable information on essential and still not fully understood aspects of the early evolution of stars and protoplanetary systems, such as the origin of jets, the interplay between the disk

* Based on observations carried out with the ESO Very Large Telescope, Cerro Paranal, Chile, under proposals 71.C-0429(C, D); and on material retrieved from the ESO Science Archive. and the stellar magnetic field, or the mechanisms governing accretion. It is thus worth devoting effort to the decoding and disentangling of the information contained in the spectra of young stellar objects.

Complete near- and mid-infrared spectral energy distributions of large quantities of young stellar objects in nearby starforming regions, obtained in the last few years with Spitzer, have produced dramatic advances in the understanding of the structure, chemistry, and evolution of circumstellar disks (e.g. Merín et al. 2008; Evans et al. 2009, and references therein). They will soon be complemented by far-infrared observations with Herschel (Dominik \& Ceccarelli 2005) and future high spatial resolution observations at millimeter and submillimeter wavelengths with ALMA (Ménard 2005; Guilloteau $\&$ Dutrey 2008). The concurrent development of sophisticated disk models (see e.g. D'Alessio et al. 2005, 2006; Dullemond \& Dominik 2005; Dullemond et al. 2007; Furlan et al. 2008; Meijer et al. 2009, for recent work) has provided a sound framework for interpreting observational results. At shorter wavelengths, 
high-resolution visible and near-infrared imaging with the Hubble Space Telescope (HST) (Stapelfeldt et al. 2003; Coffey et al. 2007; Watson \& Stapelfeldt 2007), by adaptive optics (Kóspál et al. 2008) or by spectroastrometry (Whelan et al. 2006) have helped to understand the origin of jets by probing their launch regions in the proximity of the star, allowing a comparison between the observed kinematics and spectral information with the predictions of models.

High-resolution spectroscopy in the visible and near infrared remains an essential tool for investigating jets, accretion, and winds in the immediate proximity of stellar objects, despite the general lack of direct information on the spatial distribution of the emitting medium. Permitted and forbidden lines arising from gas at widely different physical conditions provide a rich resource for investigating the density, excitation, and kinematics of the different emitting components (Appenzeller et al. 1984; Hamann 1994; Hartigan et al. 1995; Appenzeller et al. 2005), which we intend to exploit in this study.

The young stellar object Th 28 is a remarkable member of the nearby Lupus 3 region of star formation (Comerón 2008). Its visible spectrum corresponds to that of an extreme $\mathrm{T}$ Tauri star, dominated by strong emission lines on a weak continuum whose absorption features are strongly diluted by veiling (Gahm et al. 2008). It was first noted as an emission-line star together with other members of the region by Thé (1962), and it was also identified in the comprehensive $\mathrm{H} \alpha$ objective prism survey of southern star-forming regions of Schwartz (1977), from where its alternative denomination as $\mathrm{Sz} 102$ arises. Its rich emissionline spectrum and the fast-moving chain of Herbig-Haro objects that it drives, HH 228, were first noted by Krautter (1986), and the following studies have often referred to Th 28 as Krautter's star. Hughes et al. (1994) noted that the very low luminosity of the star, to which a tentative spectral type K0: was assigned, seems to come from blocking of its direct light by an edge-on disk, consistent with the fast proper motion of the jet knots at the distance of Lupus 3 when compared to their radial velocities, which were previously determined by Graham \& Heyer (1988). The base of the jet has been studied more recently with STIS at HST by Coffey et al. (2004, 2007). The spectral energy distribution of Th 28 obtained with Spitzer (Chapman et al. 2007, Merín et al. 2008) shows a rising flux toward long wavelengths indicative of large amounts of warm dust, perhaps in the form of a massive circumstellar disk. An extended envelope scattering light from the central source is directly shown by HST images, as discussed by Chapman et al. (2007). However, those authors also note that a typical signature of near-edge-on disks, a pronounced drop of flux near $8 \mu \mathrm{m}$ (D'Alessio et al. 1999), is not present in the spectral energy distribution of Th 28. The age of Th 28 cannot be estimated from its position in the temperaturemagnitude diagram given the high uncertainty in its spectral type and the likelihood that its low apparent luminosity is caused by the blocking of its light by circumstellar dust. However, the temperatures and luminosities of other young stellar objects, the ongoing star formation in Lupus 3 (Tachihara et al. 2007), and the evidence of a massive circumstellar envelope suggest a very young age for Th 28, probably not exceeding 2 Myr.

The abundance of diagnostic lines that the spectrum of Th 28 displays make it a very promising target for studying the different physical components that contribute to them, especially when kinematical information can be used to disentangle these components. In this paper we present high resolution spectroscopy of Th 28 obtained with the Very Large Telescope (VLT) that reveal a surprising variety of line profiles, and we provide tentative interpretations of their origins.

\section{Observations}

The observations described in this paper were obtained on the night of 8 to 9 June 2003 using UVES, the Ultraviolet and Visible Echelle Spectrograph at Kueyen, the second unit of the VLT. UVES has two arms optimized for observations in the blue and the red spectral ranges separately, using a dichroic to allow for simultaneous observations in the two arms. The setting that we used produced spectra centered at $\lambda=3900 \AA$ in the blue and $\lambda=5800 \AA$ in the red. The combined spectrum covers the entire wavelength range between 3300 and $6800 \AA$ using a slit 1 .'. 2 wide, which provides a resolution of $\lambda / \Delta \lambda=33000$ over this range. The exposure time was $1512 \mathrm{~s}$. The slit was oriented following the parallactic angle, which was between $13^{\circ}$ and $19^{\circ}$ away from the east-west direction during the exposure, because of the rather large distance of Th 28 from the meridian (approximately $2.5 \mathrm{~h}$ ) at the time our observations were made. In this way, not only the central object but also the nearest knot of HH 228 to the west of Th 28 was within the slit. The full-width at half maximum $(F W H M)$ of the continuum of Th 28 along the spatial direction was 1 .'3.

For the analysis we used the results of the UVES reprocessing project, which produced spectra reduced with version 3.2 of the UVES instrument pipeline for most of the point sources observed since the beginning of the operations of this instrument. Since the pipeline extracts point-source spectra, the results presented here correspond to the spectroscopic signatures found only in the immediate, unresolved surroundings of Th 28 , without a contribution from the detached knot. However, we separately extracted spectra of the knot for comparing of its velocity and FWHM.

The detailed data reduction process carried out by the UVES pipeline can be found elsewhere ${ }^{1}$ so it is only summarized here. The reduction starts by subtracting the frame bias and the interorder background. Then, the spectra at each order are extracted using optimum extraction, which removes the sky signal as well. A flat field is then extracted from the master flat field calibration frame using the same profile as for the object, and the extracted object spectrum is divided by it. Next, a dispersion solution is applied to each extracted order and a single one-dimensional spectrum is produced by merging the orders. Finally, a master response curve is applied to produce the final relative flux-calibrated spectrum. The master calibrations used in this process are certified by the ESO Data Processing and Quality Control group. Our only further processing of the spectrum produced by the pipeline was the addition of the heliocentric correction.

\section{Results}

\subsection{Adopted distance}

The distance to the Lupus 3 cloud is rather uncertain, with most values appearing in the literature lying in the $100-250 \mathrm{pc}$ range (see Comerón 2008, for a more detailed review of distance determinations in Lupus). Because they are based on the extinction jump method, most determinations agree on a distance around 140 to 160 pc (e.g. Hughes et al. 1993; Crawford 2000; Lombardi et al. 2008), which agrees with the Hipparcos distance of 140-145 pc derived by de Zeeuw et al. (1999) for the adjacent groups of the Scorpius-Centaurus association to which the

\footnotetext{
1 http://wWw . eso.org/observing/dfo/quality/reproUVES/ processing.html
} 
Lupus clouds belong. However, most of the stars used as probes in those studies are near the Lupus 1 and Lupus 4 clouds, rather than Lupus 3. Given the large angular extent of the Lupus cloud complex $\left(\sim 20^{\circ}\right)$, differences in the distances of the individual composing clouds of up to a few ten parsecs are to be expected if the extent of the complex in the radial direction is comparable to the extent in the plane of the sky. A greater distance of Lupus 3 is indeed suggested by the Hipparcos geometric parallax measurement of HR 5999 and HR 6000, the two Herbig Ae/Be stars near the center of the cloud, for which Bertout et al. (1999) find $208_{-32}^{+46} \mathrm{pc}$ and $241_{-40}^{+60} \mathrm{pc}$, respectively, as well as by the kinematic measurements of Makarov (2007) that indicate a substantial depth $(\sim 80 \mathrm{pc})$ along the line of sight and a distance near 175 pc for Lupus 3 . We have preferred to adopt a distance of $200 \mathrm{pc}$ in this study, which is consistent with the trigonometric distances found by Bertout et al. (1999), while not being too far from the overall distance generally adopted by other studies of the Lupus clouds. While being far from negligible, such uncertainty has nevertheless few consequences for the study presented here.

\subsection{Knot evolution}

Th 28 remains an active source of jets as clearly seen in images taken a few years apart, which reveal the fast proper motions of previously expelled knots and the generation of a new knot close to the central source in the intervening years. The early published observations of the Th 28/HH 228 system, together with imaging material found in the ESO archives near and before the time of our spectroscopic observations, allow us to reconstruct the recent history of jet knot ejection, measure proper motions, and determine whether knots exist near the central source. Wang \& Henning (2009) have recently carried out an analysis similar to the one that we describe here, reaching very similar conclusions.

The main knots of HH 228 were reported by Krautter (1986), who was able to measure the proper motion of the eastern one by comparing his images obtained in May 1985 with a plate taken over a decade earlier. The peak of this knot, named $\mathrm{HHE}_{1}$ (Graham \& Heyer 1988), was located 30" from Th 28 in 1985 and can be recognized on images obtained with the FORS 1 instrument at the VLT through an [SII] filter on 2 May 2003, in which the peak has shifted to $37^{\prime \prime} .7$ from the star. A second eastern node, $\mathrm{HHE}_{2}$, then lying 87" away from the star, was identified by Graham \& Heyer (1988) on images obtained on 4 June 1988. This knot is also seen in our FORS1 images, having moved away from Th 28 in the intervening 15 years to a distance of $94^{\prime \prime}$, thus confirming its association with Th 28 . The derived proper motions for $\mathrm{HHE}_{1}$ and $\mathrm{HHE}_{2}$ are $0.43 \mathrm{yr}^{-1}$ and $0.47 \mathrm{yr}^{-1}$ respectively, with an uncertainty of $0 . \mathrm{y}^{\prime} 03$.

Unfortunately, Th 28 lies at the edge of the FORS1 frame, and the western side is outside the field of view. However, the western knot identified by Krautter, HHW, is clearly visible in archive images taken with the Wide Field Imager (WFI) at the ESO-MPI $2.2 \mathrm{~m}$ telescope on La Silla on 11 July 2004, also using an [SII] filter. These were the images obtained by Wang \& Henning (2009) for their study. In those images HHW peaks at $45^{\prime \prime} .2$ from Th 28 , thus confirming its motion away from the star. Both $\mathrm{HHE}_{1}$ and $\mathrm{HHE}_{2}$ appear in those images as well, although very faint and superimposed on a faint ghost image of the nearby bright stars HR 5999/6000, making it difficult to accurately determine the positions of their peaks. The derived proper motion for HHW is similar to those of the eastern knots, 0 .' $40 \mathrm{yr}^{-1}$. That our measurement is slightly above that of Wang \& Henning (2009), despite having used the same images, reflects the uncertainty that derives from measuring proper motions on such moderately extended objects. Similar small discrepancies are found by comparing our measurements of the eastern knots with those reported by Wang \& Henning (2009).

In observations obtained in all those epochs Th 28 appears elongated in the east-west direction, coincident with the axis defined by the more distant knots. Archive HST images obtained in 1997 with the Wide Field Planetary Camera 2 (WFPC2) at the HST through the $F 555 W$ filter show a resolved core, although the resolved structure cannot be readily identified as a disk on the basis of the shape. The WFI images obtained by Wang \& Henning (2009) in 2004 show a knot located approximately 4". 5 west of Th 28, which we identify as the knot falling on the UVES slit at the time of our observations together with Th 28. This knot, to which we refer as $\mathrm{HHW}_{2}$ in the remainder of this paper, is not identified in the images published by Krautter (1986), suggesting that its birth took place after 1985 and implying a proper motion $>0.25 \mathrm{yr}^{-1}$ if it originated within a few ten $\mathrm{AU}$ from the star, consistent with a proper motion similar to those of the other knots. On the other hand, we tentatively detect a peak located 2"' 1 to the west of Th 28 on the WFPC2 image. If this is the counterpart of $\mathrm{HHW}_{2}$, the proper motion implied would be $0 . ' 34 \mathrm{yr}^{-1}$, although with a poorer accuracy $\left(\sim 0^{\prime} .05 \mathrm{yr}^{-1}\right)$ due both to the uncertainty in the precise position of the peak and to the shorter time span.

The proper motions measured for $\mathrm{HHE}_{1}, \mathrm{HHE}_{2}$, and HHW in the $0.40-0.47$ range imply velocities of $380-450 \mathrm{~km} \mathrm{~s}^{-1}$ on the plane of the sky at the adopted distance of 200 pc. Even if the lower value of $150 \mathrm{pc}$ is adopted, velocities are still in the $280-340 \mathrm{~km} \mathrm{~s}^{-1}$, which is high for Herbig-Haro objects. An intriguing feature of these measurements arises when combining them with the radial velocities of the knots, measured by Graham \& Heyer (1988). $\mathrm{HHE}_{1}$ and $\mathrm{HHE}_{2}$ have radial velocities of $-62 \mathrm{~km} \mathrm{~s}^{-1}$ and $-82 \mathrm{~km} \mathrm{~s}^{-1}$, whereas HHW is receding at only $+38 \mathrm{~km} \mathrm{~s}^{-1}$. For the new knot $\mathrm{HHW}_{2}$ near Th 28 , we measure $+25 \mathrm{~km} \mathrm{~s}^{-1}$ in our spectra. It thus appears that the radial velocities of the western knots are significantly lower than those of the eastern ones, despite their transverse velocities being similar. A bulk motion toward us of entire the system, including Th 28, seems unlikely, as it would require a systemic radial velocity of about $-20 \mathrm{~km} \mathrm{~s}^{-1}$, notably different from the average velocity of the gas in Lupus $3\left(\sim+4 \mathrm{~km} \mathrm{~s}^{-1}\right.$; Tachihara et al. 2001). Further evidence against such a high bulk velocity is presented below. A possibility is that the knots are not moving together with the gas, but that they represent instabilities in the jets through which the gas flows, as noted by Bacciotti et al. (1995), which would imply that only the radial component of the velocity reflects actual motions of the gas. Adopting $+4 \mathrm{~km} \mathrm{~s}^{-1}$ and $200 \mathrm{pc}$ as the velocity and distance of the star, respectively, and assuming that the transverse knot velocity is a lower limit to the transverse velocity of the gas, we derive a maximum angle between the axis of the jet and the plane of the sky of $5^{\circ}$, where the strongest constraint is posed by the high transverse velocity of HHW combined with its low radial velocity.

\subsection{Line profiles}

The spectrum of Th 28 is rich in both resonance and forbidden emission lines. The latter sample a wide range of excitation conditions, as shown by the coexistence in the spectrum of neutral and singly and doubly ionized oxygen, and of critical densities, from [OII] $3726\left(\sim 10^{3} \mathrm{~cm}^{-3}\right)$ to [OI] $5577\left(\sim 10^{8} \mathrm{~cm}^{-3}\right)$. We also find a remarkable variety of line profiles when observed at high spectral resolution. This variety is illustrated in Figs. 1-3, 

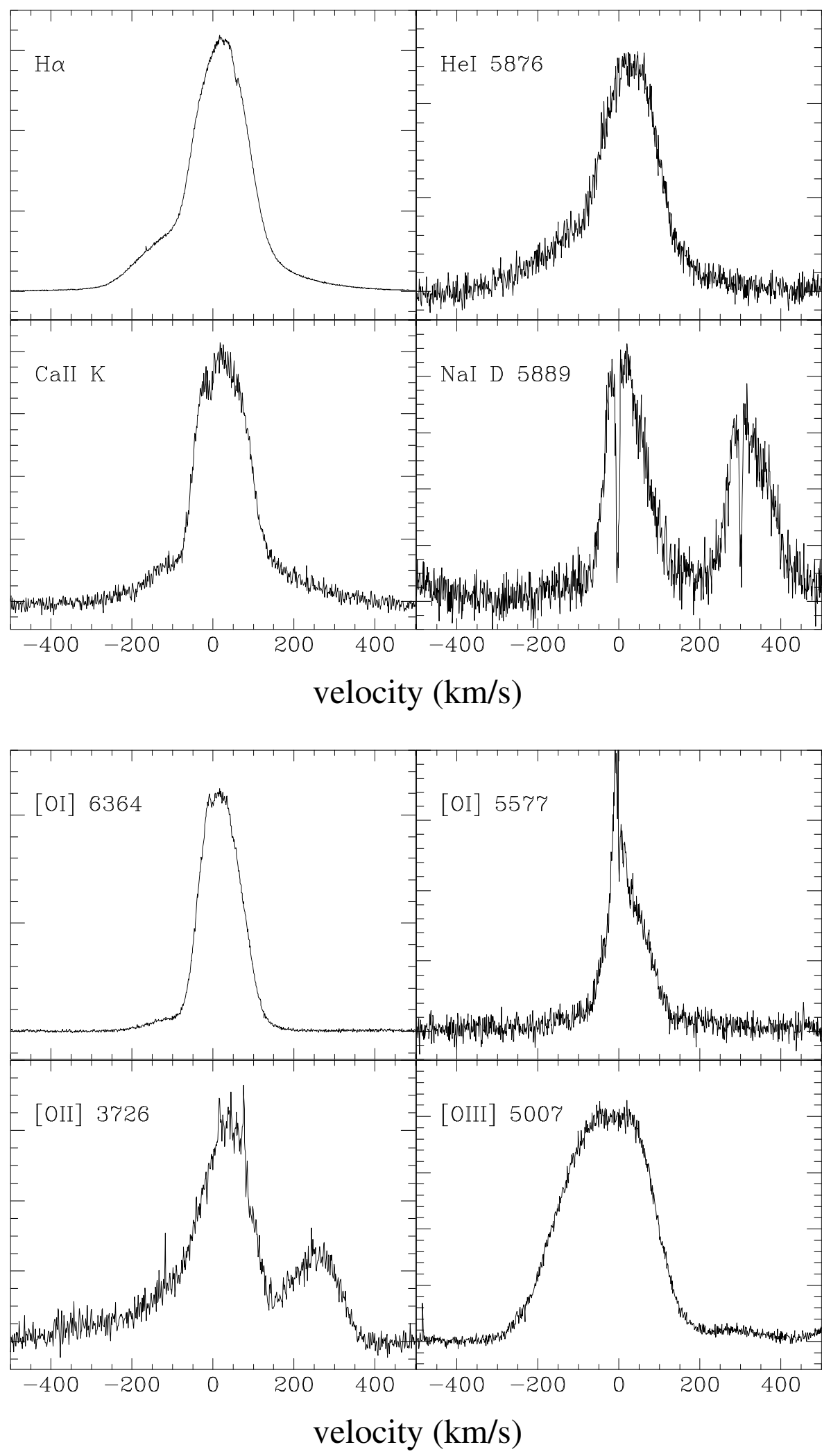

Fig. 1. Profiles of the $\mathrm{H} \alpha$, HeI 5876, CaII K, and NaI D lines. The profiles of the remaining Balmer lines is virtually identical to that of $\mathrm{H} \alpha$. The vertical axis has been adjusted to properly display each spectral line and is not representative of the relative fluxes.

Fig. 2. Profiles of the main oxygen lines, representing in the top panels two different initial levels of the [OI] atom with very different critical densities, $1.5 \times 10^{6} \mathrm{~cm}^{-3}$ for $\lambda=$ $6364 \AA$ and $9.6 \times 10^{7}$ for $\lambda=5577 \AA$. The strongest forbidden lines of singly and doubly ionized oxygen are plotted in the bottom panels. The peak to the right of the [OII] 3726 line corresponds to the [OII] 3729 line. Note the very broad profile of the [OIII] 5007 line. The sharp peak near rest velocity in the [OI] 5577 line is an artifact caused by subtraction of the strong telluric line at that position. The vertical axis has been adjusted to properly display each spectral line and is not representative of the relative fluxes.

where the velocity profiles of the lines that we discuss in this paper are presented.

A few patterns common to many of the lines can be identified:

- A broad peak with a $F W H M$ of $\simeq 130 \mathrm{~km} \mathrm{~s}^{-1}$, redshifted to $\simeq+23 \mathrm{~km} \mathrm{~s}^{-1}$ with respect to the line rest wavelength, dominates most of the lines. The broad peak can be readily identified in the Balmer lines, the forbidden lines of [NII] and [FeII], CaII K, NaII D, and HeI. A similar peak is also visible in the [OII] lines, although their peak is redshifted to $+40 \mathrm{~km} \mathrm{~s}^{-1}$

- A high-velocity blueshifted wing, which can be traced out to $300-350 \mathrm{~km} \mathrm{~s}^{-1}$ in the most intense lines, is seen in most 
Table 1. Summary of properties of the main types of emission profiles.

\begin{tabular}{lcll}
\hline \hline Feature & Velocity $\left(\mathrm{km} \mathrm{s}^{-1}\right)$ & Lines & Proposed origin \\
\hline main peak & +23 & almost all & Western jet \\
high-velocity blue wing & up to 350 & almost all & stellar wind \\
high-velocity red wing & up to 450 & only permitted lines & accretion \\
plateau & $\sim 0-5$ & [SII], [OI] 6300, [OI] 6364 & rotation? \\
\hline
\end{tabular}

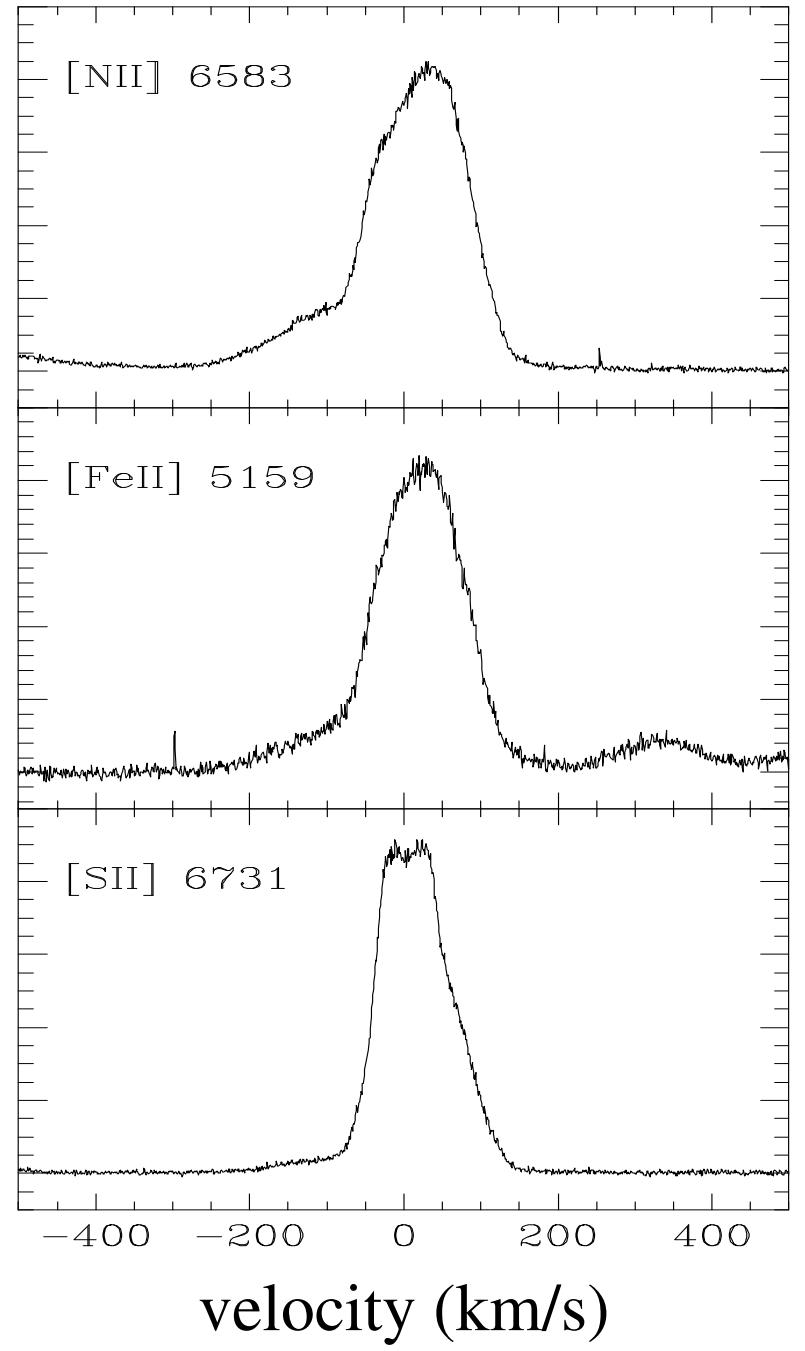

Fig. 3. Profiles of the strongest forbidden lines of [NII], [FeII], and [SII]. See also Fig. 2 for comparison with the forbidden lines of oxygen. The vertical axis has been adjusted to properly display each spectral line and is not representative of the relative fluxes.

of the lines such as the Balmer series, $\mathrm{CaII} \mathrm{K},[\mathrm{FeII}]$, HeI, [NII], [OI] (certainly at $6300 \AA$ and $6364 \AA$, and possibly also in the weaker line at $5577 \AA$ ), [OII], and [SII]. It may be weakly present too in $\mathrm{NaI} \mathrm{D}$, where the noise prevents us from detecting it beyond $-200 \mathrm{~km} \mathrm{~s}^{-1}$.

- A high-velocity redshifted wing, extending up to 400-450 $\mathrm{km} \mathrm{s}^{-1}$, is quite visible in the Balmer lines and in CaII K. Most of it can also be traced in HeI, where it becomes dominated by the noise beyond $+300 \mathrm{~km} \mathrm{~s}^{-1}$. This feature is not seen in any of the forbidden lines, or in the $\mathrm{NaI} \mathrm{D}$ lines.

- A plateau centered near the line rest wavelength appears only in the $[\mathrm{OI}]$ and $[\mathrm{SII}]$ lines.
- Finally, some lines have particular profiles where the components just described are not readily identified. The [OIII] lines at $4959 \AA$ and $5007 \AA$ are much broader than the others $\left(F W H M=270 \mathrm{~km} \mathrm{~s}^{-1}\right)$, and the [OI] line at $5577 \AA$ seems to peak near zero velocity, although this is somewhat uncertain due to the strong telluric emission at that wavelength.

The main properties of these features, together with their proposed origin as discussed in Sect. 4, are summarized in Table 1. Other lines are visible in the mid-infrared spectrum of Th 28 obtained with Spitzer, such as [NeII] at $12.814 \mu \mathrm{m}$ and [FeII] at $17.936 \mu \mathrm{m}$. Unfortunately, the resolution of the Spitzer spectra is not high enough to investigate their profiles.

\section{Discussion}

\subsection{The redshifted outflow}

The peak velocity of the dominant component of most of the spectral lines, $+23 \mathrm{~km} \mathrm{~s}^{-1}$, is comparable to the recession velocity of knot HHW (Graham \& Heyer 1988), $+38 \mathrm{~km} \mathrm{~s}^{-1}$, and is very similar to the radial velocity of $\mathrm{HHW}_{2}$ that we measure in our spectra, $+25 \mathrm{~km} \mathrm{~s}^{-1}$. It is thus likely to be associated with the origin of the outflows that produce the knots to the western side of Th 28, and also with the elongation of the image of the Th 28 central source that is common to the images obtained in all epochs (the denominated jet W in Krautter 1986). The $F W H M$ of this feature, $130 \mathrm{~km} \mathrm{~s}^{-1}$, is much broader than that of knot $\mathrm{HHW}_{2}\left(F W H M=55 \mathrm{~km} \mathrm{~s}^{-1}\right)$. Part of this broadening may be expected from the contribution to the observed flux by light scattered by dust in the circumstellar environment of Th 28 , as the outflow moves at high velocity with respect to it (Appenzeller et al. 2005). We note, however, that no asymmetry in the line profiles is clearly seen, contrary to what one may expect if the dust were largely placed closer to the star and the outflow were moving away from it, thus scattering redshifted line emission in our direction.

Although the peak is not readily identified in the profiles of the [SII] lines, we consider it likely that the outflow is the dominant contributor to their red wings, as we discuss in Sect. 4.4. The profiles of the [SII] 6716 and 6731 lines are practically identical across the whole velocity interval, whereas their ratio [SII] $6731 /$ [SII] $6716=1.76$ is somewhat below the highdensity saturation value (Osterbrock 1989) and coincident with the value expected near the critical electron density of those lines, $\simeq 3 \times 10^{3} \mathrm{~cm}^{-3}$, indicating that the [SII] emission at all velocity intervals is dominated by gas with electron densities close to critical. We estimate a high electron temperature, $T_{\mathrm{e}} \simeq 12000-13000 \mathrm{~K}$ for this component based on the [NII] 6583/[OI] 6300 ratio (Hamann 1994). This temperature is over twice as high as derived using the $\mathrm{H} \alpha /[\mathrm{NII}](6548+6583)$ ratio in Eq. (1) in Bacciotti et al. (1995), whose validity is restricted to the low ionization fraction regime $x<0.3$. This large discrepancy thus suggests that the ionization fraction in 
this component is substantially higher than 0.3 . Indeed, the temperature that we derive is closer to the estimate of Bacciotti \& Eislöffel (1999) for the parameters of jet W in Krautter (1986) ${ }^{2}$, where a high ionization fraction is also inferred. Their derived electron density is somewhat lower, which can be readily explained by their discussion being restricted to the segment of jet W lying between $2^{\prime \prime}$ and $11^{\prime \prime}$ from Th 28 , whereas the region that we consider is much closer to the central star. Based on all the above, we refer to his structure as the inner western jet.

The inner western jet is probably related to the base of the receding jet at 0.3 from Th 28 sampled by Coffey et al. (2004, 2007, 2008) using STIS observations at the HST performed in June 2004, only one year after our UVES observation. Their analysis of ultraviolet and visible forbidden lines (Coffey et al. 2008) allows them to derive average electron densities of $10^{4} \mathrm{~cm}^{-3}$, high electron temperatures $\left(T_{\mathrm{e}}=20000 \mathrm{~K}\right)$, an ionization fraction $x=0.3$, and an estimate of the mass outflow rate of $1.2 \times 10^{-8} M_{\odot} \mathrm{yr}^{-1}$. The spatially resolved spectroscopy shows significant changes in physical conditions both across and along the jet, as well as hints of jet rotation (Coffey et al. 2007).

The NaI D lines appear to be associated with this component alone. Their $F W H M, 120 \mathrm{~km} \mathrm{~s}^{-1}$, is much broader than observed in the sample of $\mathrm{T}$ Tauri stars with edge-on disks discussed by Appenzeller et al. (2005), who devote considerable attention to explaining the narrow profiles and their possible connection with their regions of origin. Even though the Th 28 disk is probably observed near to the edge-on as well, the broader profiles that we measure suggest that the narrowness of these lines is not entirely ubiquitous among such systems.

\subsection{A stellar wind}

The distinct blueshifted wing that appears in most of the lines under study, described in Sect. 3.3, is most likely the signature of a weakly collimated or uncollimated tenuous, hot, and highly ionized stellar wind (Ferreira et al. 2006). The association with a low-density medium is supported by the appearance of this signature in most forbidden lines, including those with low critical densities like [OII] 3726 and 3729, [SII] 6716 and 6731, or [NII] 6548 and 6583. The profile of the HeI 5876 line, where this high-velocity, blueshifted component is clearly seen, fully agrees with the results of Beristain et al. (2001) and Appenzeller et al. (2005), who conclude that HeI emission is not restricted to the proximities to the surface of the star where high temperatures are produced at the base of magnetospheric accretion columns, but that it can also arise in hot outflows, as is the case in Th 28. The high excitation of the wind is also consistent with the weakness of the blue wing of the low excitation species like NaI, [SII], and $[\mathrm{OI}]$.

The stellar wind is the only structural component of the circumstellar environment of Th 28 whose density and excitation conditions are suitable for the production of [OIII] emission, explaining the unique profiles of the lines at 4959 and $5007 \AA$ (Fig. 2). The poor collimation of the wind causes their very large FWHM, whereas the blueshift of its intensity may be the consequence of the obscuration of the half of the wind ejected toward the western hemisphere by a circumstellar disk. Such obscuration suggests that only the regions of the wind closest to the star, which can be easily blocked from view by a circumstellar disk,

\footnotetext{
2 Bacciotti et al.'s observations of jet W were obtained in 1987, two years after the original observations of Krautter (1986), and thus refer essentially to the same structure.
}

emit enough intensity for detection. Additional indications of the close proximity of the wind emission region to the surface of the star comes from the Balmer lines, all of which have nearly identical profiles and lack the self-absorption features that are otherwise frequently observed in the spectra of T Tauri stars (Edwards et al. 1994; Hartmann et al. 1994; Reipurth et al. 1996), except for a weak absorption centered near $+60 \mathrm{~km} \mathrm{~s}^{-1}$ and visible only in $\mathrm{H} \alpha$ thanks to the high signal-to-noise ratio in that line. Such absorption features are caused when two or more structural components with different physical conditions overlap kinematically along the line of sight. Their virtual absence in the Balmer lines of Th 28 indicates that the stellar wind has too low a density in the region where such overlap with the inner western jet occurs from our vantage point. The closer proximity of the wind emission region to the star is also consistent with a circumstellar disk blocking its western side, but not the inner western jet.

\subsection{Accretion}

The absence of an extended red tail in any of the forbidden lines, as noted in Sect. 3.3, clearly indicates that this feature visible in most of the resonance lines arises in a very highdensity medium, under the conditions that are found near the surface of the star, thus being most likely related to magnetospheric accretion. Additional evidence of accretion comes from the previously known strong CaII triplet emission, whose line intensities are measured to be approximately in the proportion 1:1.2:1 (Comerón et al. 2003), indicating emission in an optically thick medium. The high temperature of the dense gas contributing to this emission component is revealed by the relatively strong HeI emission and by the undetectability of emission in the NaI D lines, most probably the result of a high ionization faction of sodium.

If indeed caused by accretion, the most extreme velocities in the long redshifted tail should be indicative of the terminal velocity of infalling gas at the base of the accretion columns, which is approximately the free-fall velocity (Muzerolle et al. 1998a). The tail can be traced in $\mathrm{H} \alpha$ out to a velocity $v=+450 \mathrm{~km} \mathrm{~s}^{-1}$, implying $M / R=v^{2} / 2 G \simeq 0.42 M_{\odot} R_{\odot}^{-1}$, where $M$ and $R$ are the mass and radius of the star, respectively. Such high free-fall velocity argues against a very low mass for Th 28. Assuming an age of $2 \mathrm{Myr}$ for Th 28 , this $M / R$ ratio is attained at a mass $M=0.6 M_{\odot}\left(D^{\prime}\right.$ Antona \& Mazzitelli 1997), with a surface temperature $T_{\text {eff }}=4100 \mathrm{~K}$ (which would correspond to a main spectral type approximately K7; Kenyon \& Hartmann 1995) and an intrinsic luminosity $L=0.5 L_{\odot}$. The large infrared excess and strong outflow activity suggest an even younger age, implying higher mass, temperature, and luminosity. For $1 \mathrm{Myr}$, the corresponding values are $M=0.9 M_{\odot}, T_{\text {eff }}=4600 \mathrm{~K}$ (spectral type $\mathrm{K} 4$ ), and $L=1.7 L_{\odot}$. From the temperature and luminosity, and using the bolometric corrections from Kenyon \& Hartmann (1995), we estimate an intrinsic magnitude $I_{C}$ between 11.2 (if the age is $2 \mathrm{Myr}$ ) and 10.0 (for an age of $1 \mathrm{Myr}$ ). Despite the considerable uncertainty, this is much brighter than the observed $I_{C}=15.1$ (Comerón et al. 2003), whereas the observed colors are similar to those of an unreddened star, which we take as an indication that we see the Th 28 central star mainly in scattered light, as already suggested by Hughes et al. (1994).

The $I_{C}$ intrinsic magnitude, together with the radius of the star obtained from $L$ and $T_{\text {eff }}$, allows us to derive the flux emitted by the star per unit area in the CaII triplet lines, which has been shown to be closely correlated with the accretion 
rate $^{3}$ (Muzerolle et al. 1998b; Mohanty et al. 2005; Nguyen et al. 2009). Using the equivalent width $E W(\mathrm{CaII} 8662)=$ $25 \AA$ measured by Comerón et al. (2003) for the CaII line at $8662 \AA$, we can then estimate the accretion rate as $\log \dot{M}_{\text {acc }}=$ $1.06 \log \left(F_{\text {CaII } 8662}\right)-15.40$ (Mohanty et al. 2005), where $\dot{M}_{\text {acc }}$ is given in $M_{\odot} \mathrm{yr}^{-1}$ and $F_{\text {CaII }} 8662$ is the emitted flux in that line per unit surface of the star. In this way we obtain an estimated $\dot{M}_{\text {acc }} \sim 4.2 \times 10^{-8} M_{\odot} \mathrm{yr}^{-1}$ when taking the parameters for an age of 2 Myr discussed above, or $\dot{M}_{\text {acc }} \sim 6.3 \times 10^{-8} M_{\odot} \mathrm{yr}^{-1}$ for $1 \mathrm{Myr}$.

When compared to the estimated mass, the $\dot{M}_{\text {acc }} / M$ ratio places Th 28 somewhat above the average $\dot{M}_{\text {acc }} \propto M^{2}$ relation (e.g. Mohanty et al. 2005; Luhman et al. 2007), but well within the scatter found among other $\mathrm{T}$ Tauri stars of similar mass. However, it is surprising that our estimate for the accretion rate is similar to the mass outflow rate at the base of the receding jet alone derived by Coffey et al. (2008) (Sect. 4.1), whereas outflow-to-accretion ratios among $\mathrm{T}$ Tauri stars are commonly found to be in the 0.01-0.1 range (Hartigan et al. 1995; Coffey et al. 2008). The result needs to be taken with caution given the very indirect and rough way in which we estimated both the mass and the accretion rate, in addition to the scatter in the average relationship between CaII triplet emission and accretion (Herczeg \& Hillenbrand 2008), and our assumption that CaII triplet emission is dominated by accretion. Furthermore, the calculation of the mass outflow rate in the jet also has its own uncertainties, as described in Coffey et al. (2008). Finally, both accretion and mass loss rates are usually variable, while the observations discussed here, on which each of these quantities is estimated, are not simultaneous. We thus cannot rule out a true average accretion rate substantially higher than the one estimated above or a lower average outflow rate, although most probably not by as much as two orders of magnitude for either. In a qualitative sense, we take our results as an indication that the $\dot{M}_{\text {outflow }} / \dot{M}_{\text {acc }}$ ratio is on the high side for Th 28 .

\subsection{The [SII] and [OI] lines}

Whereas the shape of most of the emission lines in the spectrum of Th 28 can be explained in terms of the three structural components discussed in the previous sections (the inner western jet, the uncollimated stellar wind, and the accretion flow), the [SII] and [OI] lines have particular profiles that do not entirely fit this scheme. The [SII] lines at $6716 \AA$ and $6731 \AA$ and the [OI] lines of relatively low critical density at $6300 \AA$ and $6364 \AA$ are characterized by nearly flat-top shapes (Figs. 2 and 3). On the other hand, although the precise location of the peak of the [OI] line at $5577 \AA$ is uncertain because of the contamination of the extracted spectrum by the much stronger telluric line at the same wavelength, it does not seem to be located near $+23 \mathrm{~km} \mathrm{~s}^{-1}$, as is the case in other lines where the emission that we associate with the inner western jet dominates.

A detailed examination of the [SII] lines (Fig. 3) shows that their red tail is very similar to that of other forbidden lines and in general to the red tail of the emission that we associate with the inner western jet. However, as we move bluewards we find a rather sudden change of slope that becomes much steeper at around $+40 \mathrm{~km} \mathrm{~s}^{-1}$ until the plateau is reached at $\sim+35 \mathrm{~km} \mathrm{~s}^{-1}$. On the blue side, the plateau ends at $-30 \mathrm{~km} \mathrm{~s}^{-1}$ and is followed

${ }^{3}$ The implicit assumption is that the CaII triplet lines come entirely from accretion. Unfortunately we cannot confirm that this is the case from the line profiles, as they fall outside the spectral range of our highresolution observations.

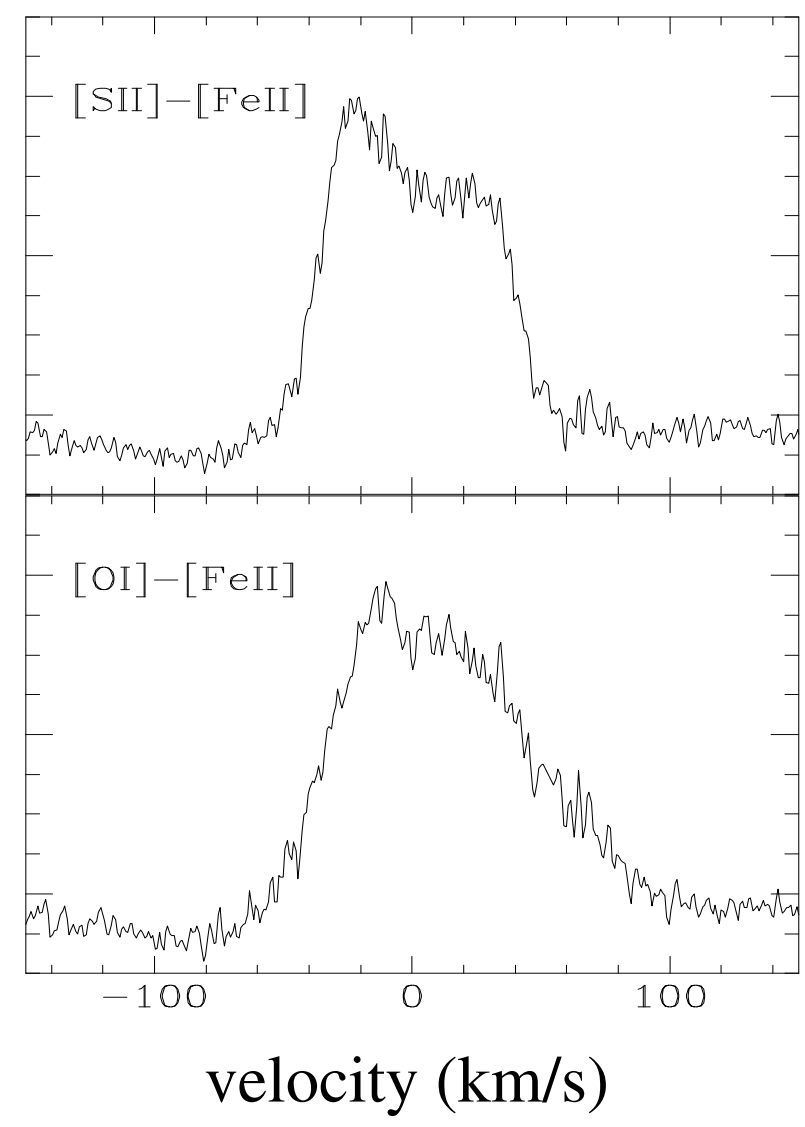

Fig. 4. Residual profiles of the [SII] 6731 (top) and [OI] 6364 (bottom), after subtraction of the profile of the [FeII] 5159 shifted to the same velocity interval and scaled by a factor so as to remove the contribution of the redshifted outflow (Sect. 4.1). The slight depression to the right of the features is caused by subtraction of the extended blue wing of the $[\mathrm{FeII}]$ line, which is proportionally more intense than the blue wings of the [SII] and [OI] lines. The contribution of the redshifted outflow is well removed from the spectra of the [SII] lines, but it cannot be removed from the $[\mathrm{OI}]$ lines at velocities below $100 \mathrm{~km} \mathrm{~s}^{-1}$.

by a similarly steep slope. This suggests that the plateau in the [SII] lines stems from a structurally distinct component, centered on $+2 \mathrm{~km} \mathrm{~s}^{-1}$, which is very similar to the velocity of the molecular gas in the region and probably reflects the true radial velocity of Th 28 .

To better isolate this component, we subtracted the [FeII] 5159 line from the [SII] 6731 line, after scaling the former by a factor chosen so as to minimize the red wing of the [SII] line that we attribute to formation in the inner western jet. We caution the reader that there is no physical basis underlying this approach, since the conditions of the medium from which these lines arise, hence probably their regions of origin as well, are different. However, the similarity of the component of the line shape that we attribute to the inner western jet among the different lines, despite their different critical densities and excitation conditions, suggests that it is possible to define a "typical" line shape associated with this component, which we use here for reference and which is represented by the profile of the [FeII] 5159 line. As the top panel of Fig. 4 shows, by following this procedure, it is possible to completely cancel out the red wing in the difference and thus isolate the component contributing to the plateau. Since subtracting of the scaled [FeII] profile removes flux in the range of velocities covered by the plateau, and taking into account that the inner western jet emission peaks 
near the red end of the plateau, the [FeII]-subtracted [SII] profile is not a plateau anymore, but rather a two-peaked line where the blue peak is more prominent than the red one.

We tried the same procedure to subtract the contribution of the inner western jet from the profile of the [OI] 6300 and 6364 lines. However, in this case we find that no complete cancellation of the flux redwards of $+40 \mathrm{~km} \mathrm{~s}^{-1}$ is possible, unlike in the case of the [SII] lines, and only cancellation beyond $100 \mathrm{~km} \mathrm{~s}^{-1}$ is achieved. On the other hand, a blue peak appears at a velocity similar to (albeit slightly lower than) that of the blue peak of the [SII] lines. A gentle decrease in flux then follows towards more positive velocities until the end of the plateau is reached, although the drop in flux just beyond either side of the plateau is not as abrupt as in the [SII] residual lines. Our interpretation is that most of the residual flux in the [OI] lines appears to be associated with essentially the same region where the [SII] residual flux originates. However, different physical conditions in the regions of the redshifted outflow where the [OI] and [FeII] lines are produced may be the cause of an imperfect subtraction of the contribution of the redshifted outflow from the [OI] profile, thus diluting the double-peaked profile limited by sharp drops in flux that is most clearly seen in the [SII] residual profiles. The residual flux of the [OI] 6300 and 6364 lines after subtraction appears to be actually similar to the [OI] 5577 line, suggesting that, despite the different of two orders of magnitude in their respective critical densities, both sets of lines may arise from the same component, with the caveat already expressed above that the profile of the [OI] 5577 line at low velocities is severely contaminated by telluric emission.

Regardless of the strict validity of the procedure outlined above based on the subtraction of the scaled [FeII] 5159 line profile, the plateau at the center of the [SII] and [OI] lines flanked by a sharp drop in flux is suggestive of rotation in the region where this component is formed. The plateau profile might also arise in principle from the combination of emissions from the two components of a bipolar jet moving in opposite directions and the broader component attributed to the inner western jet filling the flux between the two peaks. However, the sharp drop in intensity beyond the edges of the plateau suggests that the emission from each jet component should be intense and cover a very narrow ranges of velocities, and the intensity near zero velocity due to jet emission should then drop to very near zero, thus resulting in a clearly two-peaked profile very different from the actually observed plateau. We instead favor rotation as the main reason for the observed shape of this component of the [SII] lines ${ }^{4}$. Although indications of rotation in the western jet of Th 28 have been reported by Coffey et al. (2007), we note that the profile of the [SII] and [OI] lines presented by those authors is markedly different from the one observed by us, suggesting that the line profile discussed here arises closer to the star than the region 0.3 away from it $(\simeq 70 \mathrm{AU}$ at the assumed distance of $200 \mathrm{pc}$ ) sampled by the STIS observations of Coffey et al. (2007).

\footnotetext{
${ }^{4}$ We note here that the residual profile of the [SII] line, including the asymmetry between peaks, is very similar to the profile of the [SII] lines of Par-Lup3-4, another low-luminosity object in Lupus 3 possessing a small jet whose discovery we reported in Fernández \& Comerón (2005). In that paper we used the small separation in velocity between the peaks to argue that the Par-Lup3-4 jet must be moving in a direction close to the plane of the sky, assuming that the doubly-peaked line arose in oppositely moving jets. However, the results presented here call into question that interpretation, as the structure of the [SII] lines of ParLup3-4 may also be dominated by rotation, rather than by gas moving radially away from the star.
}

We thus propose an origin of the residual component in a different region from the jets. As already noted, the plateau structure is most clearly seen in the [SII] lines, and it can be discerned in the [OI] 6300 and 6364 lines. It is also hinted at by the shape of the [NII] 6583 line (Fig. 3), where it appears as a hump centered on $-30 \mathrm{~km} \mathrm{~s}^{-1}$. This suggests that the emission arises in a region of density not much higher than $\sim 10^{3} \mathrm{~cm}^{-3}$, near the critical density of the [SII] lines. The emitting region may be an extended disk atmosphere co-rotating with the disk. If this is the case, assuming pure Keplerian circular rotation and a viewing geometry of the emitting region close to edge-on, the difference between the velocities at the edge of the plateau and its center corresponds to the circular velocity in the innermost orbit. Taking $32 \pm 5 \mathrm{~km} \mathrm{~s}^{-1}$ as the value of that difference and an estimated mass between $0.6 M_{\odot}$ and $0.9 M_{\odot}$ discussed in Sect. 4.3, the inner edge of the emitting structure is estimated to be placed at $\sim 0.7 \pm 0.4 \mathrm{AU}$ from the central star. The location of this region roughly corresponds to the jet launch region favored in the modeling of Ferreira et al. (2006). The asymmetry between the peaks at the edge of the plateau cannot be explained by a differentially rotating, axially symmetric emitting region. We speculate that such asymmetries may be caused by a nonaxisymmetric disk (Watson \& Stapelfeldt 2007), perhaps due to transient phenomena associated with regional heating of the circumstellar disks by X-ray flares (Ercolano et al. 2008; Meijerink et al. 2008), as Th 28 is known to be a relatively strong X-ray emitter (Gondoin 2006). It should be pointed out as well that a strong departure from axial symmetry is implied by the analysis of line ratios across the resolved width of the Th 28 jet carried out by Coffey et al. (2008). Finally, there are marked differences between the profiles of the [SII] lines reported here and those in the spectra of Appenzeller et al. (1984), where there are no hints of a flat-top profile. This leaves the possibility open that the $[\mathrm{SII}]$ and perhaps also the [OI] profiles are dominated by transient structures.

\section{Conclusions}

In this paper we have used a variety of emission lines in the spectrum of Th 28 to infer some fundamental aspects of its immediate circumstellar environment, such as the main structural components responsible for the emission. Our main conclusions can be summarized as follows.

- When considering observations obtained at different epochs to study the mass outflow of Th 28, special attention needs to be paid to the fast evolution of its jets. We present evidence showing the formation of a new knot near Th 28 between the time of the first identification of the Herbig-Haro companions to Th 28 in 1985 and the time of our observations, as well as new measurements of the motions of previously known knots based on observations separated by almost two decades. The measurements confirm the fast motion of the knots projected on the plane of the sky.

- There is considerable diversity among the line profiles, both permitted and forbidden, testifying to the variety of physical environments where they originate.

- Most of the lines are dominated by a moderately broad redshifted component peaking at $+23 \mathrm{~km} \mathrm{~s}^{-1}$. It is likely that this component corresponds to the collimated outflow that in the past generated the currently detached knots observed to the west of Th 28.

- We present evidence of an uncollimated stellar wind characterized by high temperature and excitation, as shown by 
its emission in $\mathrm{HeI}$ and [OIII]. The [OIII] lines display a remarkably broad profile and appear to have their origin in this component alone. The appearance of a blueshifted wing in the HeI line associated with this component confirms that HeI emission in young stellar objects can originate in outflows, in addition to accretion.

- Evidence is also found for an accretion flow in the form of an extended red wing in the profile of several permitted lines. The origin of this wing in a high-density medium is demonstrated by its absence in all the forbidden lines. The red wing can be traced up to $+450 \mathrm{~km} \mathrm{~s}^{-1}$. Assuming that this is the free-fall terminal velocity at the surface of the star, the suggested mass is $0.6-0.9 M_{\odot}$. The infall rate is estimated to be $\sim 4.2-6.3 \times 10^{-8} M_{\odot} \mathrm{yr}^{-1}$ from published equivalent widths of the CaII infrared triplet lines, within the expected range for T Tauri stars. However, this is surprisingly low in comparison with the published estimate of a mass outflow of $1.2 \times 10^{-8} M_{\odot} \mathrm{yr}^{-1}$ in the receding jet alone. Given the large uncertainties in the computation of both quantities, we take this as an qualitative indication of a large mass outflow/mass inflow ratio in Th 28 .

- An additional component different from those discussed above seems to significantly contribute to the flux in the [SII] lines, as well as in the [OI] lines. The flat-top shape of the [SII] lines suggests that this component arises in a lowdensity rotating medium that we tentatively associate with a disk atmosphere. Assuming Keplerian rotation, the velocity range spanned by this component suggests that the disk has a central hole of $\sim 0.7 \pm 0.4$ AU radius.

Some ingredients still need to be analyzed to obtain a full picture of the immediate circumstellar environment of Th 28 , such as its mid-infrared spectral energy distribution in order to better understand the structure and viewing geometry of the circumstellar disk. However, the data presented here represent important probes of the region where the interplay between stellar winds, disk winds, the bases of jets and accretion flows takes place.

Acknowledgements. We are pleased to thank the staff of Paranal Observatory Science Operations for the execution of this programme in Service Mode at the VLT, and to the User Support Department of the ESO Data Management and Operations Division for their careful revision of the Phase 2 package defining our observations. We thank Dr. Bruno Merín for making the Spitzer spectra of Th 28 available to us, and the anonymous referee for constructive comments leading to several improvements in this paper. We gratefully acknowledge the effort made by the ESO Data Management and Operations and Software Development Divisions in developing the UVES data reduction pipeline and in making the pipeline-reprocessed data publicly available through the ESO Science Archive Facility.

\section{References}

Appenzeller, I., Jankovics, I., \& Östreicher, R. 1984, A\&A, 141, 108 Appenzeller, I., Bertout, C., \& Stahl, O. 2005, A\&A, 434, 1005 Bacciotti, F., \& Eislöffel, J. 1999, A\&A, 342, 717

Bacciotti, F., Chiuderi, C., \& Oliva, E. 1995, A\&A, 296, 185 Beristain, G., Edwards, S., \& Kwan, J. 2001, ApJ, 551, 1037 Bertout, C., Robichon, N., \& Arenou, F. 1999, A\&A, 352, 574 Chapman, N. L., Lai, S.-P., Mundy, L. G., et al. 2007, ApJ, 667, 288
Coffey, D., Bacciotti, F., Woitas, J., Ray, T. P., \& Eislöffel, J. 2004, ApJ, 604, 758

Coffey, D., Bacciotti, F., Ray, T. P., Eislöffel, J., \& Woitas, J. 2007, ApJ, 663, 350

Coffey, D., Bacciotti, F., \& Podio, L. 2008, ApJ, 689, 1112

Comerón, F. 2008, The Lupus clouds, in Handbook of Star Forming regions, ed. B. Reipurth, ASP Monographs, 2

Comerón, F., Fernández, M., Baraffe, I., Neuhäuser, R., \& Kaas, A. A. 2003, A\&A, 406, 1001

Crawford, I. A. 2000, MNRAS, 317, 996

D’Alessio, P., Calvet, N., Hartmann, L., Lizano, S., \& Cantó, J. 1999, ApJ, 527, 893

D’Alessio, P., Merín, B., Calvet, N., Hartmann, L., \& Montesinos, B. 2005, RMxA\&A, 41, 61

D’Alessio, P., Calvet, N., Hartmann, L., Franco-Hernández, R., \& Servín, H. 2006, ApJ, 638, 314

D’Antona, F., \& Mazzitelli, I. 1997, Mem. Soc. Astron. It., 68, 807

de Zeeuw, P. T., Hoogerwerf, R., de Bruijne, J. H. J., Brown, A. G. A., \& Blaauw, A. 1999, AJ, 117, 354

Dominik, C., \& Ceccarelli, C. 2005, in The dusty and molecular universe: a prelude to Herschel and ALMA, ed. A. Wilson, ESA SP-577

Dullemond, C. P., \& Dominik, C. 2005, A\&A, 424, 971

Dullemond, C. P., Hollenbach, D., Kamp, I., \& D'Alessio, P. 2007, in Protostars and Planets V, ed. B. Reipurth, D. Jewitt, \& K. Keil (Univ. of Arizona Press) Edwards, S., Hartigan, P., Ghandour, L., \& Andrulis, C. 1994, AJ, 108, 1056

Ercolano, B., Drake, J., Raymond, J. C., \& Clarke, C. C. 2008, ApJ, 688, 398

Evans, N. J., Dunham, M. M., Jørgensen, J. K., et al. 2009, ApJS, 181, 321

Ferreira, J., Dougados, C., \& Cabrit, S. 2006, A\&A, 456, 785

Fernández, M., \& Comerón, F. 2005, A\&A, 440, 1119.

Furlan, E., McClure, M., Calvet, N., et al. 2008, ApJS, 176, 184

Gahm, G. F., Walter, F. M., Stempels, H. C., Petrov, P. P., \& Herczeg, G. J. 2008, A\&A, 482, L35

Gondoin, P. 2006, A\&A, 454, 595

Graham, J. A., \& Heyer, M. H. 1988, PASP, 100, 1529

Guilloteau, S., \& Dutrey, A. 2008, ApSS, 313, 95

Hamann, F. 1994, ApJS, 93, 485

Hartigan, P., Edwards, S., \& Ghandour, L. 1995, ApJ, 452, 736

Hartmann, L., Hewett, R., \& Calvet, N. 1994, ApJ, 426, 669

Herczeg, G. J., \& Hillenbrand, L. A. 2008, ApJ, 681, 594

Hughes, J., Hartigan, P., \& Clampitt, L. 1993, AJ, 105, 571

Hughes, J. H., Hartigan, P., Krautter, J., \& Kelemen, J. 1994, AJ, 108, 1071

Kenyon, S. J., \& Hartmann, L. 1995, ApJS, 101, 117

Kóspál, Á., Ábrahám, P., Apai, D., et al. 2008, MNRAS, 383, 1015

Krautter, J. 1986, A\&A, 161, 195

Lombardi, M., Lada, C. J., \& Alves, J. 2008, A\&A, 480, 785

Luhman, K. L., Joergens, V., Lada, C., et al. 2007, in Protostars and Planets V, ed. B. Reipurth, D. Jewitt, \& K. Keil (Univ. of Arizona Press)

Makarov, V. V. 2007, ApJ, 658, 480

Meijer, J., Waters, L. B. F. M., de Koter, A., et al. 2009, A\&A, 496, 741

Meijerink, R., Glassgold, A. E., \& Najita, J. 2008, ApJ, 676, 518

Ménard, F. C. 2005, The Cool Universe: Observing Cosmic Dawn, ed. C. Lidman, \& D. Alloin, ASP Conf. Ser., 344

Merín, B., Jørgensen, J., Spezzi, L., et al. 2008, ApJS, 177, 551

Mohanty, S., Basri, G., \& Jyawardhana, R. 2005, Astron. Nachr., 326, 891

Muzerolle, J., Calvet, N., \& Hartmann, L. 1998a, ApJ, 492, 743

Muzerolle, J., Hartmann, L., \& Calvet, N. 1998b, AJ, 116, 455

Nguyen, D. C., Scholz, A., van Kerkwijk, M. H., Jayawardhana, R., \& Brandeker, A. 2009, ApJ, 694, L153

Osterbrock, D. 1989, Astrophysics of Gaseous Nebulae and Active Galactic Nuclei (Univ. Science Books)

Reipurth, B., Pedrosa, A., \& Lago, M. T. V. T. 1996, A\&AS, 120, 229

Schwartz, R. D. 1977, ApJS, 35, 161

Stapelfeldt, K. R., Ménard, F., Watson, A. M., et al. 2003, ApJ, 589, 410

Tachihara, K., Toyoda, S., Onishi, T., et al. 2001, PASJ, 53, 1081

Tachihara, K., Rengel, M., Nakajima, Y., et al. 2007, ApJ, 659, 1382

Thé, P. S. 1962, Contrib. Bosscha Obs., 15

Wang, H., \& Henning, Th. 2009, AJ, 138, 1072

Watson, A. M., \& Stapelfeldt, K. R. 2007, AJ, 133, 845

Whelan, E. T., Ray, T. P., Bacciotti, F., \& Jayawardhana, R. 2006, NewAR, 49, 582 